\title{
Towards a bioengineered uterus: bioactive sheep uterus scaffolds are effectively recellularized by enzymatic preconditioning
}

Arvind Manikantan Padma ${ }^{1,2}$, Laura Carrière (iD ${ }^{1,2}$, Frida Krokström Karlsson ${ }^{1,2}$, Edina Sehic ${ }^{1,2}$, Sara Bandstein ${ }^{1,2}$, Tom Tristan Tiemann ${ }^{1,2,3}$, Mihai Oltean ${ }^{1,4}$, Min Jong Song ${ }^{1,2,5}$, Mats Brännström ${ }^{1,2,6}$ and Mats Hellström (iD ${ }^{1,2 \bowtie}$

Uterine factor infertility was considered incurable until recently when we reported the first successful live birth after uterus transplantation. However, risky donor surgery and immunosuppressive therapy are factors that may be avoided with bioengineering. For example, transplanted recellularized constructs derived from decellularized tissue restored fertility in rodent models and mandate translational studies. In this study, we decellularized whole sheep uterus with three different protocols using $0.5 \%$ sodium dodecyl sulfate, $2 \%$ sodium deoxycholate (SDC) or $2 \%$ SDC, and $1 \%$ Triton X-100. Scaffolds were then assessed for bioactivity using the dorsal root ganglion and chorioallantoic membrane assays, and we found that all the uterus scaffolds exhibited growth factor activity that promoted neurogenesis and angiogenesis. Extensive recellularization optimization was conducted using multipotent sheep fetal stem cells and we report results from the following three in vitro conditions; (a) standard cell culturing conditions, (b) constructs cultured in transwells, and (c) scaffolds preconditioned with matrix metalloproteinase 2 and 9. The recellularization efficiency was improved short-term when transwells were used compared with standard culturing conditions. However, the recellularization efficiency in scaffolds preconditioned with matrix metalloproteinases was $200-300 \%$ better than the other strategies evaluated herein, independent of decellularization protocol. Hence, a major recellularization hurdle has been overcome with the improved recellularization strategies and in vitro platforms described herein. These results are an important milestone and should facilitate the production of large bioengineered grafts suitable for future in vivo applications in the sheep, which is an essential step before considering these principles in a clinical setting.

npj Regenerative Medicine (2021)6:26; https://doi.org/10.1038/s41536-021-00136-0

\section{INTRODUCTION}

Infertility, caused by an absent or dysfunctional uterus was considered incurable until our group succeeded with the world's first uterus transplantation that resulted in live birth ${ }^{1}$. This procedure has now been repeated at multiple centers resulting in more reported births ${ }^{2-9}$. Uterus transplantation is still considered an experimental treatment procedure with protocols continuously being evaluated, i.e., organ preservation protocols ${ }^{10}$, positioning of the vascular anastomoses ${ }^{11,12}$, and the use of robotic-assisted surgery to reduce recovery time and blood loss ${ }^{13-15}$. So far, almost all successful uterus transplantation cases have used live donors. However, the risks entailed by live donor surgery and the negative side-effects caused by the required immunosuppressive treatment in the allograft recipient opens up discussions for an alternative donor source.

An attractive organ option which would overcome some of these limitations is a developed bioengineered uterus from an appropriate scaffold together with the patient's own cells. Extracellular matrix (ECM) derived scaffolds proved successful in several bioengineering studies on female reproductive tissues ${ }^{16-20}$. Recently, ECM-derived uterus scaffolds were created by decellularization for the mouse ${ }^{21}$, the rat ${ }^{22-26}$, the rabbit ${ }^{27}$, the pig $^{28}$, and more recently, the sheep ${ }^{29,30}$. Furthermore, a series of successful studies on rodents from multiple independent groups suggest that decellularized uterine tissue patches can be grafted to repair a fullthickness uterine wall injury and restore fertility ${ }^{21,24,26,31}$. These successful results mandate a translational evaluation using larger animal models. We therefore developed decellularization protocols for the generation of sheep uterus scaffolds as the first step in this process $^{30}$. The sheep is by many considered the most suitable preclinical large animal model for uterine studies because of its physiological and anatomical resemblance to the human uterus, and the sheep model played a significant part in the lead up to the successful human uterus transplantation cases $^{32,33}$.

However, a major obstacle using decellularized tissue for bioengineering applications is the limited ability to recellularize the constructs and achieve an appropriate cell density which seems to play an important role in the treatment outcome. For example, recellularized constructs seem to better stimulate tissue regeneration, recruit host cells and prevent rapid scaffold degeneration compared to scaffolds without cells, in particular for larger grafts ${ }^{34-37}$.

We herein studied three different types of sheep uterus scaffolds created by decellularization, and assessed their ability to stimulate growth and angiogenesis. We further describe novel strategies how to significantly increase the recellularization

\footnotetext{
${ }^{1}$ Laboratory for Transplantation and Regenerative Medicine, Sahlgrenska Academy, University of Gothenburg, Gothenburg, Sweden. ${ }^{2}$ Department of Obstetrics and Gynecology, Clinical Sciences, Sahlgrenska Academy, University of Gothenburg, Gothenburg, Sweden. ${ }^{3}$ Department of Gynecology and Obstetrics, University Hospital of Heidelberg, Heidelberg, Germany. ${ }^{4}$ Department of Surgery, Clinical Sciences, Sahlgrenska Academy, University of Gothenburg, Gothenburg, Sweden. ${ }^{5}$ Department of Obstetrics and Gynecology, Yeouido St. Mary's Hospital, The Catholic University of Korea, Seoul, Republic of Korea. ${ }^{6}$ Stockholm IVF-EUGIN, Hammarby allé 93 , Stockholm, Sweden. ${ }^{\circledR}$ email: mats. hellstrom@gu.se
} 
efficiency. This study is therefore an important in vitro milestone toward sheep uterus bioengineering in vivo.

\section{RESULTS}

\section{Sheep uterus scaffold generation}

Whole sheep uterus organs were decellularized using three earlier established protocols ${ }^{30}$. Based on microscopic analyses, we were able to reproduce the successful protocols as no donor cells were observed. Protocol 1 (P1) derived scaffolds based on sodium dodecyl sulfate (SDS) appeared denser and demonstrated a superior morphological ECM fiber structure compared with protocol 2 (P2) generated scaffolds that was based on sodium deoxycholeate (SDC) or to scaffolds produced by protocol 3 (P3) that was based on a combination of SDC and Triton X-100. Higher magnification in the deeper layer of the myometrium compartment in scanning electron microscopy on cross sectioned dUTDs revealed more abundant collagen bundles in P1 and P2 derived scaffolds, while scaffolds generated with P3 seemed more porous (Fig. 1A-L).

\section{Scaffold bioactivity induced axon regeneration}

The bioactivity of the decellularized sheep uteri was assessed using the fetal dorsal root ganglion (DRG) assay that detects neurogenesis. It was evident that the decellularized sheep uterus tissue discs (dUTDs) displayed functional growth-promoting qualities that stimulated more axon regeneration to longer distances compared to collagen alone (Fig. 1M-Q).

\section{Scaffold preconditioning with MMPs}

To evaluate if improved cell attachment and scaffold porosity could be established to increase future scaffold recellularization efficiencies, we preconditioned dUTDs with matrix metalloproteinases (MMPs) 2 and 9. Morphologically, the tissue became more porous after the MMPs treatment and there were more distinctly stained elastic fibers after the preconditioning. Few other differences were observed in Russel-Movat pentachrome stained sections before and after MMPs treatment (Fig. 2A). Importantly, the MMPs treatment did not affect the vascular patency in large uterus scaffolds, as confirmed by vascular perfusion (Supplementary videos 1-3). Scaffold content of donor DNA, total protein, and hydroxyproline were also unaffected from the preconditioning procedure (Fig. 2B-D). The actual decellularization process did not affect the collagen fiber thickness. However, the MMPs treatment reduced it by $30-40 \%$ in all three scaffold types (Fig. 2E). Immunohistochemistry for collagen I, collagen IV, laminin, elastin, and fibronectin showed that these molecules were well retained
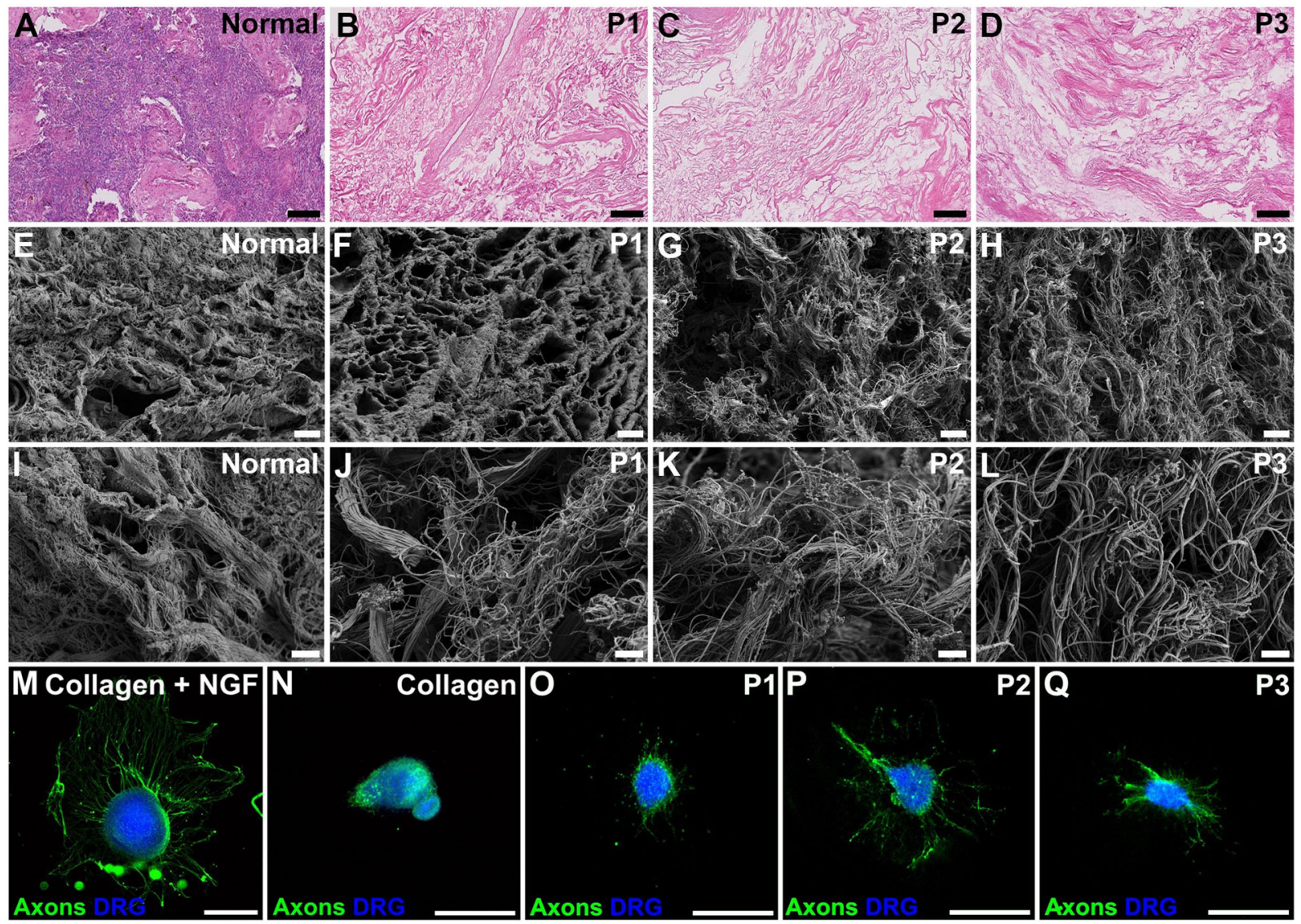

Fig. 1 Morphological and bioactivity assessment of developed sheep uterus scaffolds. Hematoxylin and eosin-stained sections from normal and decellularized uterus showed that the extracellular matrix was well-preserved and that all hematoxylin positive nuclei had been removed by the different decellularization protocols $(\mathbf{A}-\mathbf{D}$; scale bar $=100 \mu \mathrm{m})$. Scanning electron microscopy pictures obtained at $\times 3000$ magnification $\left(\mathbf{E}-\mathbf{H}_{\text {; scale }} \mathrm{bar}=8 \mu \mathrm{m}\right)$ showed that protocol $(\mathrm{P}) 1$ generated scaffolds resulted in an organized porous structure $(\mathbf{F})$ while $\mathrm{P} 2$ and P3 derived scaffolds resulted in a more compact arrangement with less organized fiber structure. Higher magnification ( $\times 12,000 ; \mathbf{I}-\mathbf{L} ;$ scale $\mathrm{bar}=2 \mu \mathrm{m})$ showed that collagen bundles remained intact in all three scaffold types. The dorsal root ganglion (DRG) assay and the chorioallantioc membrane (CAM) assay showed that all scaffolds were functionally bioactive (M-V). The DRGs regenerated axons in wells coated with collagen that was given nerve growth factor supplement in the culture medium (positive control; $\mathbf{M}$ ), but not when this growth factor was omitted (negative control, $\mathbf{N}$ ). Scaffolds derived from the three different decellularization protocols all stimulated axonal regeneration $(\mathbf{O}-\mathbf{Q}$; scale bar $=500 \mu \mathrm{m})$. 


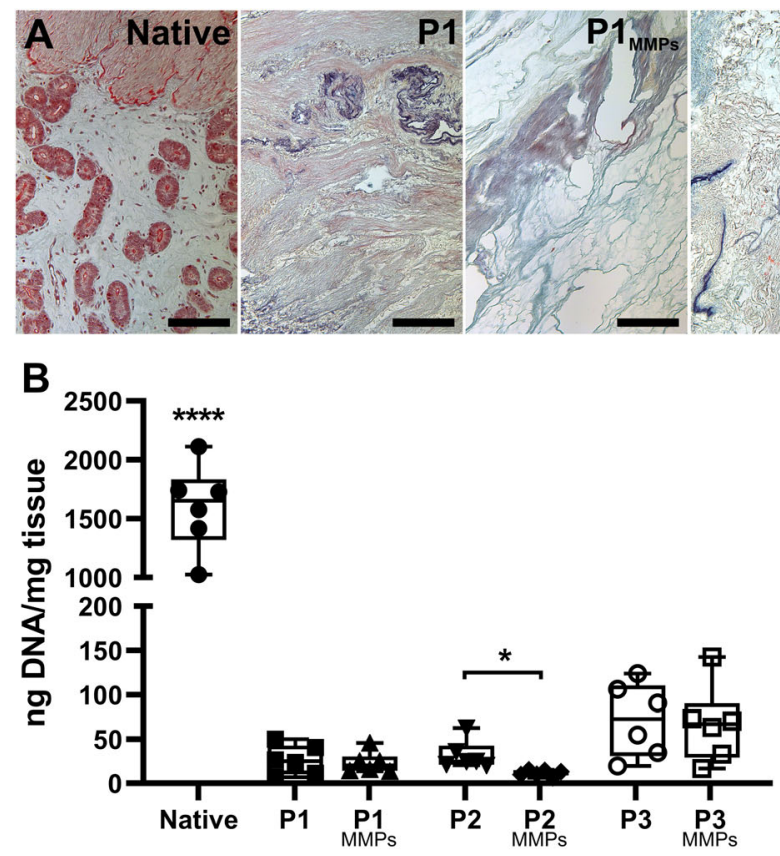

D

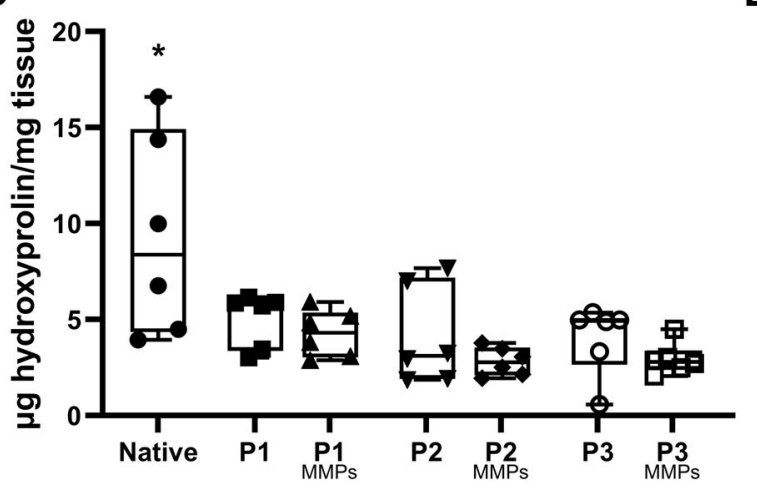

P2

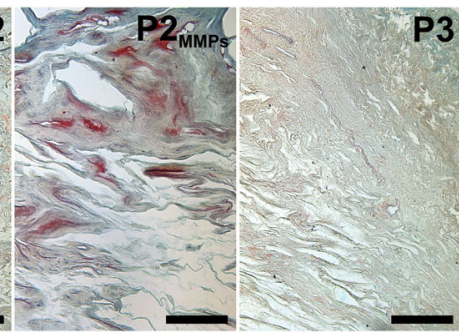

C

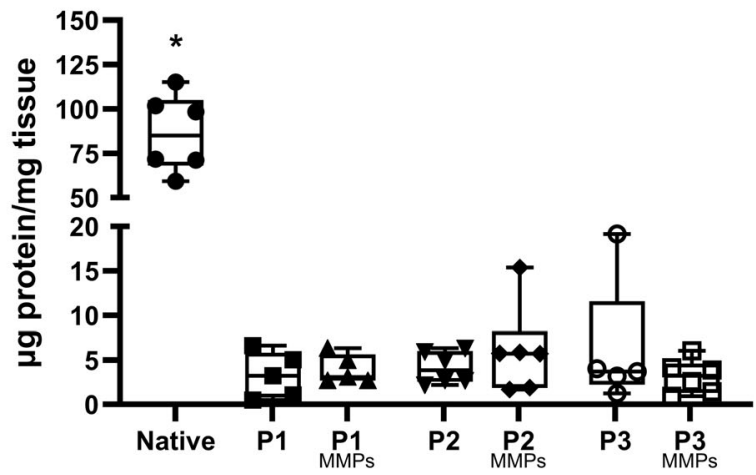

E

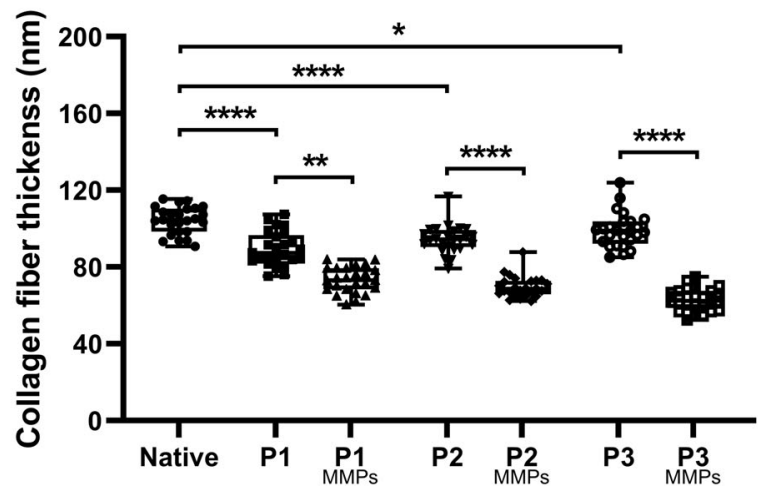

Fig. 2 The extracellular matrix composition of developed scaffolds was assessed. A Russell-Movat pentachrome stains collagen and reticular fibers (yellow), nuclei and elastic fibers (black), sulfated glycosaminoglycans (blue), muscle tissue (red), and fibrin intense red. Stained from scaffolds revealed that the matrix metalloproteinase 2 and 9 (MMPs) treatment resulted in a more porous scaffold and that elastic fibers became more distinct. Scale bars $=100 \mu \mathrm{m}$. B Each decellularization method resulted in scaffolds with very small amounts of remaining donor DNA, and that the MMPs were able to reduce this even more in protocol (P) 2 derived scaffolds. C The total protein content and $\mathbf{D}$ the hydroxyproline content were unaffected by the MMPs treatment while $\mathbf{E}$ the collagen fiber thickness was significantly reduced. Graphs show box plot with each sample value, median $\pm \mathrm{IQR}$, and range.

in the scaffolds, but elastin had aggregated to specific areas within the scaffolds after the enzymatic treatment (Fig. 3).

\section{Confirmed angiogenic bioactivity in all scaffold types}

The scaffolds were able to actively stimulate angiogenesis, evident by the CAM assay. Each scaffold type resulted in a near doubling of blood vessels in the vicinity of the inoculated decellularized uterus tissue when compared with the inert control reference point that does not stimulate any extra angiogenesis (Fig. 4). There was no difference in angiogenic effects between any of the uterus scaffold types before and after MMPs treatment.

\section{Cell characterization of SF-SCs}

Heterogeneous female sheep fetal bone marrow stem cells (SF-SC) were isolated from sheep fetus (Fig. 5A) and characterized for multipotency. The isolated SF-SCs were cuboidal and elongated under phase contrast microscope and were positive for the mesenchymal and stromal markers CD166 and vimentin (VIM), a-smooth muscle cell actin (SMA), and for estrogen receptor- $\beta 1$ (ER- $\beta$; not shown). The multipotency was demonstrated by the differentiation into chondrogenic lineage with alcian blue positive cell clusters, osteogenic lineage (positive for dentin matrix acidic phosphoprotein 1, DMP and receptor activator of nuclear factor $\mathrm{K}$ $B, R A N K$ ), and myogenic lineage (positive for SMA and myoblast determination protein 1, MyoD1; Fig. 5).

\section{Recellularization efficiency using standard conditions}

Cell counts from constructs recellularized with SF-SCs using standard conditions $\left(\mathrm{RC}_{\mathrm{sC}}\right)$ showed that the cell densities had significantly increased between day 3 and day 14 in all scaffold types (Table 1; Fig. 6). Sheep uterus scaffolds produced by P1 and P2 stimulated a greater cell proliferation during the extended cell culture period compared with scaffolds produced by P3 (Table 2). However, cells remained close to the injection site in all scaffold types and cell migration was only limited to include the superficial layers of the uterus scaffolds (Fig. 6A, D). 


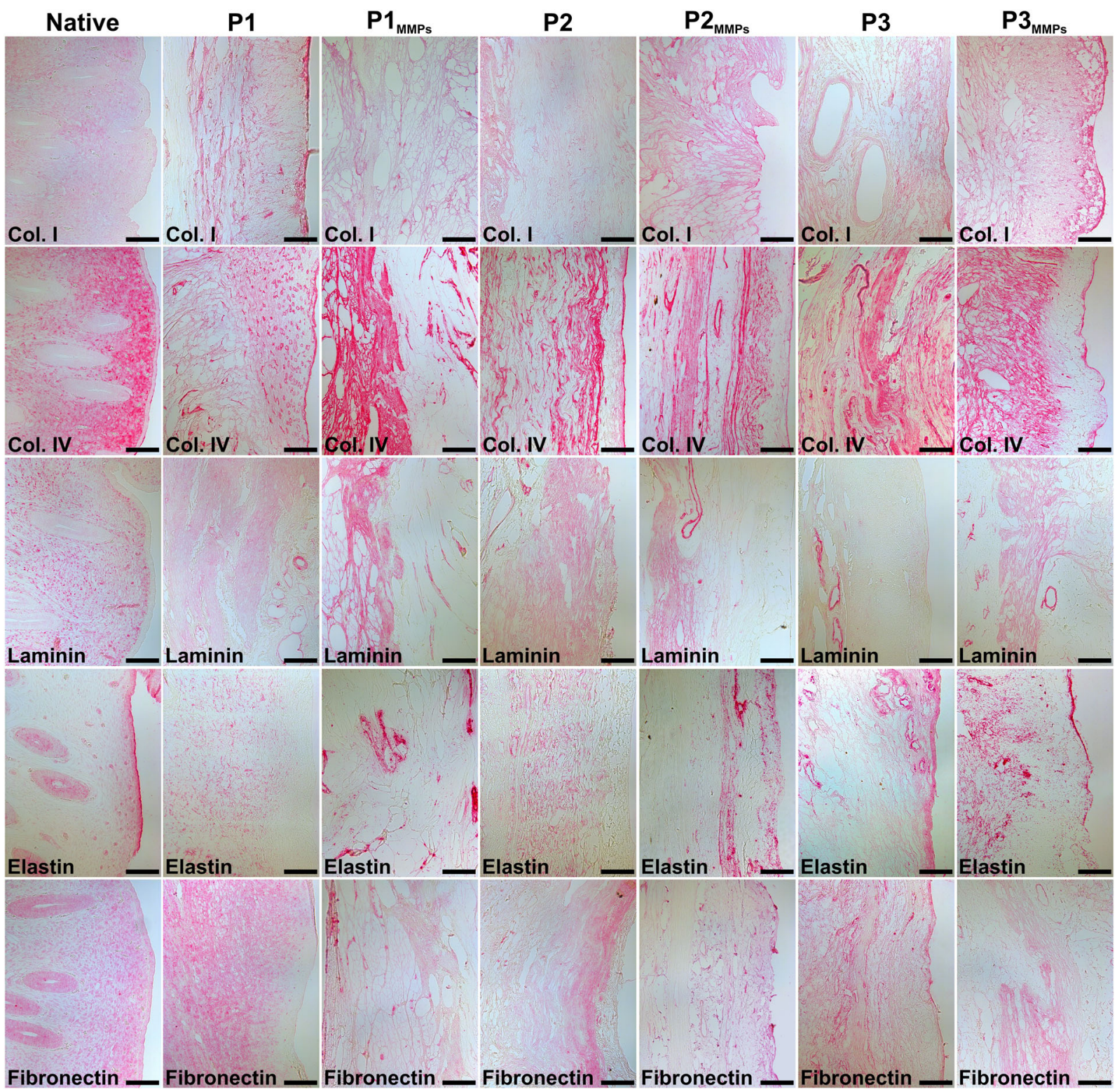

Fig. 3 Distribution of importantant structural components after scaffold production. Immunohistochemistry stained sections from decellularized sheep uterus tissue before and after matrix metalloproteinase 2 and 9 (MMPs) preconditioning, including native uterus tissue for comparison. P protocol, Col. collagen. Scale bars $=100 \mu \mathrm{m}$.

\section{Improved short-term recellularization efficiency using transwells}

To improve culture medium diffusion over the scaffold in vitro, recellularized constructs were cultured in transwell inserts $\left(R C_{T W}\right)$. Significantly more cells colonized P1 and P2 derived scaffolds compared with P3-derived scaffolds after 3 days in transwells (Table 2). This method was also better than $\mathrm{RC}_{\mathrm{SC}}$ group after three days for $\mathrm{P} 1$ and $\mathrm{P} 2$ derived constructs $(\mathrm{P} 1, p=0.0074 ; \mathrm{P} 2, p=$ 0.0226 ) which represented a cell density increase of $229 \%$ and $185 \%$, respectively. For P3-derived constructs, the efficiency increased by $278 \%$ using transwells (P3, $p=0.0504$; Fig. 6J-L). P3, $p=0.050$; Fig. $6 \mathrm{~J}-\mathrm{L}$ ). Interestingly, there was no benefit using transwells when constructs were cultured for 14 days in vitro, except for P3-derived scaffolds ( $p=0.0316$; Fig. 6M-O). While we only detected a modest long-term cell density increase using transwells, we did observe improved cell migration using this method. Cells were found further away from the injection site compared with the $\mathrm{RC}_{\mathrm{SC}}$ group (Fig. 6A, B).

\section{Enzymatic pre-treatment enhanced the recellularization efficiency}

Indeed, preconditioned scaffolds with MMPs prior to the recellularization and subsequent transwell culture $\left(\mathrm{RC}_{\mathrm{MMP}+\mathrm{TW}}\right)$ dramatically improved both cell density and cell distribution compared with the other two recellularization methods, which was further prominent after 14 days in vitro (Table 1; Fig. 6C, F). The significant increase in the recellularization efficiency after three days compared with $\mathrm{RC}_{\mathrm{SC}}$ was $221 \%, 285 \%$, and $363 \%$ for $\mathrm{P} 1$, $\mathrm{P} 2$, and $\mathrm{P} 3$, respectively (P1, $p=0.0293 ; \mathrm{P} 2, p=0.0021 ; \mathrm{P} 3, p=$ 0.0002 ; Fig. $6 \mathrm{~J}-\mathrm{L}$ ). The recellularization efficiency after 14 days in vitro and was significantly higher compared with 3e days after recellularization (Table 1). The improved cell density was also higher compared with $\mathrm{RC}_{\mathrm{SC}}$ for all scaffold types $(\mathrm{P} 1, p=0.0108$; $\mathrm{P} 2, p=0.0010 ; \mathrm{P} 3, p<0.0001)$, and compared to methods using transwells without enzymatic pre-treatment for P1 $(p=0.0125)$ and P2 constructs $(p=0.0016)$, but not for the P3 constructs (Fig. $6 \mathrm{M}-\mathrm{O})$. Many cells were found in the peripheral parts of the 

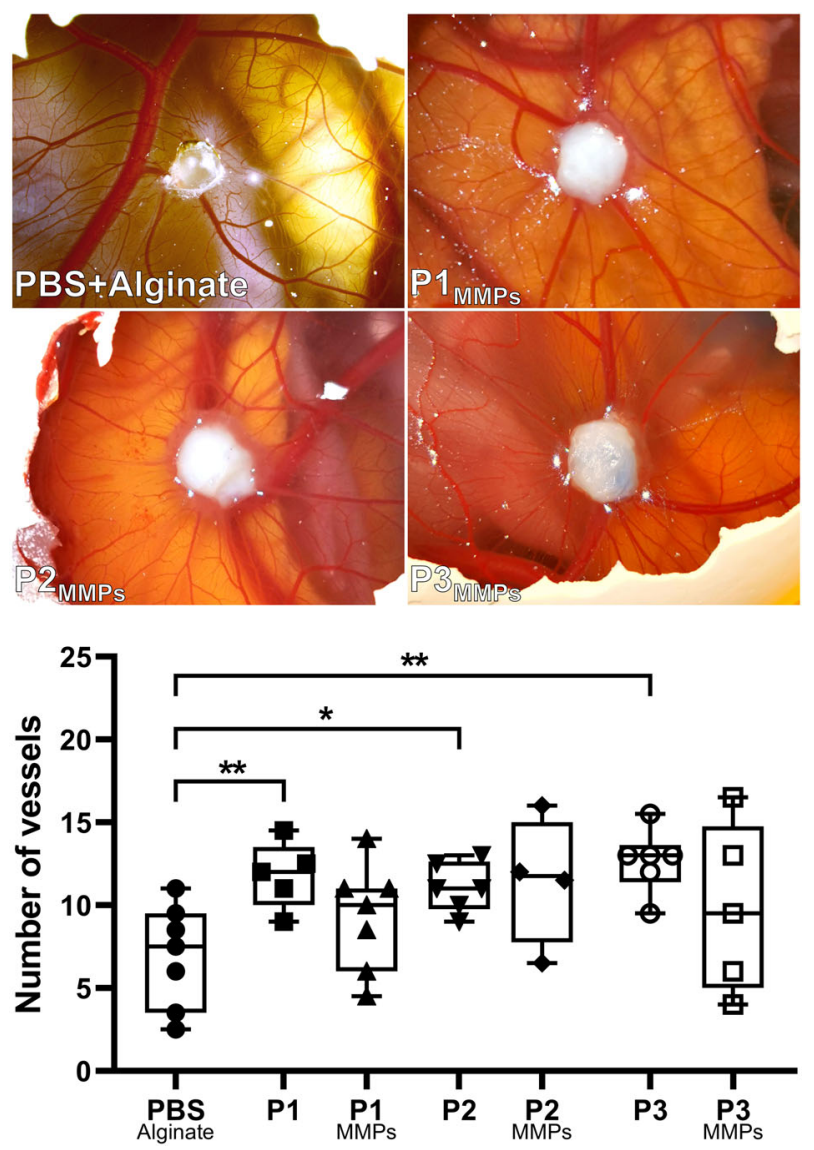

Fig. 4 The chorioallantioc membrane (CAM) bioactivity assay confirmed a significant increase in angiogenesis after the inoculation of the three scaffold types before the matrix metalloproteinase 2 and 9 (MMPs) preconditioning compared with the inert alginate reference point that indicate normal chick fetal angiogenesis. The bioactivity from the scaffolds stimulated a doubling of blood vessel formation, while there was a modest (nonsignificant) reduction in angiogenic effects after the MMPs treatment. Graphs show box plot with each sample value, median \pm IQR, and range. P protocol. Significant levels; ${ }^{*} p<0.05 ;{ }^{* *} p<0.01$.

constructs and it was evident that the enzymatic pre-treatment facilitated cell distribution across all scaffold compartments and generated better constructs than the other recellularization methods evaluated (Fig. $6 \mathrm{~A}-\mathrm{C}$ ). The cells within the scaffolds remained positive for the stem cell phenotypic markers CD166, ER- $\beta, a-S M A$, and VIM during culture, and kept their proliferative ability, evident by Ki67 positive staining (Fig. 6G-I).

\section{DISCUSSION}

In initial studies on rat uterus bioengineering, we showed that scaffolds could be created by decellularization using SDC, DMSO, and Trirton $X-100^{22}$. Simultaneously, two other groups reported that SDS or a hydrostatic pressure system could be used for the same purpose $\mathrm{e}^{24,26}$. Together with transplantation studies, multiple reports clearly demonstrated successful bioengineering strategies using decellularized material to restore fertility in rodents with a uterine injury ${ }^{21,23,31,38}$. Similar whole uterus decellularization techniques were recently also developed and evaluated in vitro with tissues from larger animal models, e.g., the rabbit ${ }^{27}$, the pig $^{28}$, and the sheep ${ }^{29,30}$.

However, it is evident that these scaffolds are difficult to recellularize efficiently. Thus, before conducting bioengineering studies in vivo on large animal models which will require significantly larger bioengineered constructs for engraftment, detailed assessments on scaffold quality and efficient recellularization methods need to be established since these factors play an essential role in graft functionality ${ }^{34,39}$. Effective recellularization of scaffolds depends on multiple factors, including the scaffold's bioactivity and structural porosity that allow culture medium and cells to migrate across the construct during the reconstruction. We therefore investigated the functional bioactivity of three sheep uterine scaffolds and assessed their ability to stimulate growth and angiogenesis. We further assessed various methods to facilitate media access and construct porosity during recellularization with the hypothesis that they may improve cellular distribution across the construct and improve recellularization.

The first bioactivity test we conducted was the DRG assay. This method clearly demonstrated that all three decellularized uterus scaffolds stimulated axonal growth. This assay did not discriminate what specific growth factors were responsible for the beneficial neuronal sprouting. However, neurons are dependent on similar cell signaling growth pathways as other cell types ${ }^{40}$ and the positive effect seen on the DRGs therefore suggests the existence of beneficial cues for uterine tissue regeneration. For example, Hiraoka et al. showed that uterine tissue regeneration was interleukin- 6 dependent after decellularized tissue was grafted in the mouse model ${ }^{21}$. This anti-inflammatory cytokine also acts as a powerful growth inducer in DRGs ${ }^{41}$. Nevertheless, additional studies will be required to determine what functional growth factors remained in the decellularized uterine tissue. However, this is complex since most straight-forward protein quantification methods are based on the detection of denatured protein fragments (i.e., non-functional proteins). In addition, our results also show that the scaffolds were free from detrimental remnants related to the decellularization procedures since it would be expected that neurons fail to initiate axonal sprouting if there were any remaining toxic decellularization chemicals ${ }^{42}$.

Similar to the DRG assay, the CAM assay confirmed functional bioactivity. This method determined scaffold-induced angiogenesis during the chicken embryo development and showed that our scaffolds stimulated a doubling of blood vessel formation on the CAM compared with normal angiogenesis during chicken fetus development, exemplified by the inert reference point used in the control group. Angiogenic activity will potentiate repair and reduce ischemic graft injury following engraftment. On the other hand, it still remains to be determined whether the effects seen are due to biologically active cues, or are due to physical/ mechanical properties of the scaffolds ${ }^{43}$. The angiogenic effect was only mildly affected by the MMPs treatment. Histologically, we noticed that the MMPs treatment had affected the elastic fiber composition and the collagen fiber thickness while leaving the hydroxyproline content stable. Importantly, these structural changes did not affect the patency of the scaffolds' vascular conduits. Hence, MMPs can be used as a scaffold preconditioning strategy in future experiments for developing larger constructs where vascular perfusion might be needed during whole organ recellularization and scaffold survival after engraftment (i.e., vascular anastomosis).

The physical properties of the scaffolds also affect cell attachment and recellularization. Recellularization is a challenging feat and few studies using decellularized tissue showed good cell densities. Furthermore, many bioengineering studies on assorted organs were transplanted after a relatively short time after recellularization ${ }^{36,44-46}$, including our own study in the rat $^{31}$. In the latter study, we also reported that the cellular reconstruction is decellularization protocol-dependent and that it affected the outcomes in vivo. However, the choice of employed recellularization strategy also matters, concerning modes of cell delivery ${ }^{47}$, time cultured in vitro ${ }^{48}$ and type of in vitro platform used (e.g., various bioreactors or spinner flasks) ${ }^{10,49-51}$. 

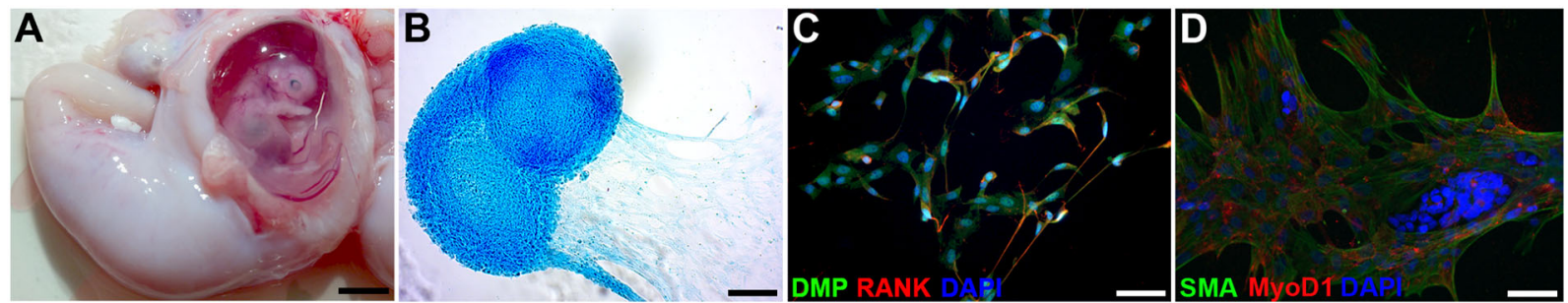

Fig. 5 Pluripotent cells used for the recellularization. Heterogeneous sheep fetal stem cells (SF-SCs) were isolated from a 6-8-week-old sheep fetus (A). These cells were expanded in vitro and their pluripotency was demonstrated after their differentiation into a chondrogenic lineage (alcian blue positive clusters; B), an osteogenic lineage positive for dentin matrix acidic phosphoprotein 1 (DMP1) and receptor activator of nuclear factor $\kappa \mathrm{B}$ (RANK; C), and a myogenic lineage positive for smooth muscle cell actin (SMA) and myoblast determination protein 1 (MyoD1; D). Scale bars; $5 \mathrm{~cm}$ (A), and $100 \mu \mathrm{m}$ (B-D).

\begin{tabular}{|c|c|c|c|c|c|c|c|c|c|c|c|}
\hline & \multicolumn{3}{|c|}{ Standard condition } & & \multicolumn{3}{|c|}{ Transwell } & & \multicolumn{3}{|c|}{$\mathrm{MMP}+$ transwell } \\
\hline & MED & MED & Difference & & MED & MED & Difference & & MED & MED & Difference \\
\hline P1 & 112.8 & 328.7 & $p=0.0003$ & P1 & 258.9 & 273.8 & n.s. & $\mathrm{P} 1$ & 250.1 & 461.6 & $p=0.0012$ \\
\hline
\end{tabular}

Hence, the experiments of the present study also aimed to evaluate novel in vitro strategies that may benefit recellularization. We first recellularized sheep uterus scaffolds using standard cell culturing conditions (the $\mathrm{RC}_{\mathrm{SC}}$ group) which represents commonly employed recellularization strategies ${ }^{28,30,31}$. The results were comparable to earlier observations; cells were sparse and concentrated near the injection site and failed to migrate within the scaffolds.

We then evaluated an alternative in vitro setting, where recellularized scaffolds rested on a permeable membrane (a transwell), which would facilitate culture medium access (the $\mathrm{RC}_{\mathrm{TW}}$ group). Surprisingly, this only provided a short-term benefit compared with the $\mathrm{RC}_{\mathrm{SC}}$ group. Nevertheless, histological analysis indicated that cells had migrated better with this method, a result congruent with that of a study on kidney scaffold recellularization $^{52}$.

We then assessed whether recellularization was improved further by the MMP2 and MMP9 preconditioning. We selected these enzymes because of their ability to specifically break down ECM components and because they are highly co-expressed by proliferative and migratory cells ${ }^{53,54}$ and can expedite angiogenesis ${ }^{55}$. The MMP pre-treatment was coupled with transwell cultures $\left(\mathrm{RC}_{\mathrm{MMP}+\mathrm{Tw}}\right.$ group), and the significant advantage was observed already after 3 days. Hence, this enzymatic preconditioning facilitated cell attachment. The effect was even more profound after 14 days when all constructs were colonized by several magnitudes higher cell numbers than after the other recellularization methods evaluated herein. Importantly, these results also showed that MMP treatment improved the least effective scaffold type (P3) to become as good as P1 and P2 derived scaffolds. Since no significant differences were seen between constructs after the enzymatic treatment, we suggest that a general reconditioning event took place that improved scaffold quality irrespective of decellularization protocol used. Furthermore, histology and scanning electron microscopy showed improved cell migration and distribution within all constructs after enzymatic preconditioning.

Naturally, the choice of cell type also plays a significant role in recellularization. It is likely necessary to use a combination of different cell types for bioengineering of large and more complex tissues than evaluated herein. However, we choose to use SF-SCs in this study that is a heterogeneous multipotent stroma cell population which could become valuable for future in vivo studies. A similar cell type was shown to produce important ECM components and growth factors that stimulate regeneration ${ }^{53}$. The SF-SCs continued to show markers for multipotentency and should thus still be able to activate valuable repair mechanisms after engraftment, e.g., as documented previously with multipotent stroma cells ${ }^{56}$ and in endometrial repair studies ${ }^{57}$. Future recellularization strategies may also include the use of hydrogels to prevent cell leakage after injection ${ }^{47}$, the use hypoxic in vitro conditions $^{28}$, alternative preconditioning protocols, and more advanced cell culturing platforms.

In summary, this study demonstrated that our decellularized tissues were functionally bioactive and stimulated important growth pathways including angiogenesis. Preconditioning the decellularized tissue with MMPs before recellularization increased cell density by $200-300 \%$ compared with standard culturing techniques and improved the cell distribution, independent of the decellularization method used for scaffold generation. Hence, our findings suggest that decellularization protocol-dependent deficiencies may be compensated for by enzymatic preconditioning. In addition, the recellularization platform and culturing technique play important roles in order to obtain cell-rich bioengineered constructs suitable for in vivo applications.

\section{METHODS}

\section{Uterus isolation and decellularization}

Eighteen sheep uteri of about $35 \mathrm{~g}$ each were isolated from 8- to 12month-old Swedish Finull sheep at a local abattoir. Since the animals were processed for food production, no prior animal welfare evaluation was required for the organ procurement. Each uterus was cannulated, flushed, and decellularized by perfusion through the organ vasculature according to three previously characterized and published protocols ${ }^{30}$. In brief, P1 was based on the decellularization detergent SDS (0.5\%), P2 on SDC (2\%), and P3 on a combination of SDC (2\%) and Triton X-100 (1\%). Each detergent in $\mathrm{P} 1$ and $\mathrm{P} 2$ was perfused for $8 \mathrm{~h}$, respectively, which was then followed up by the perfusion of deionized water (DW; $26 \mathrm{~h}$ ), phosphate 

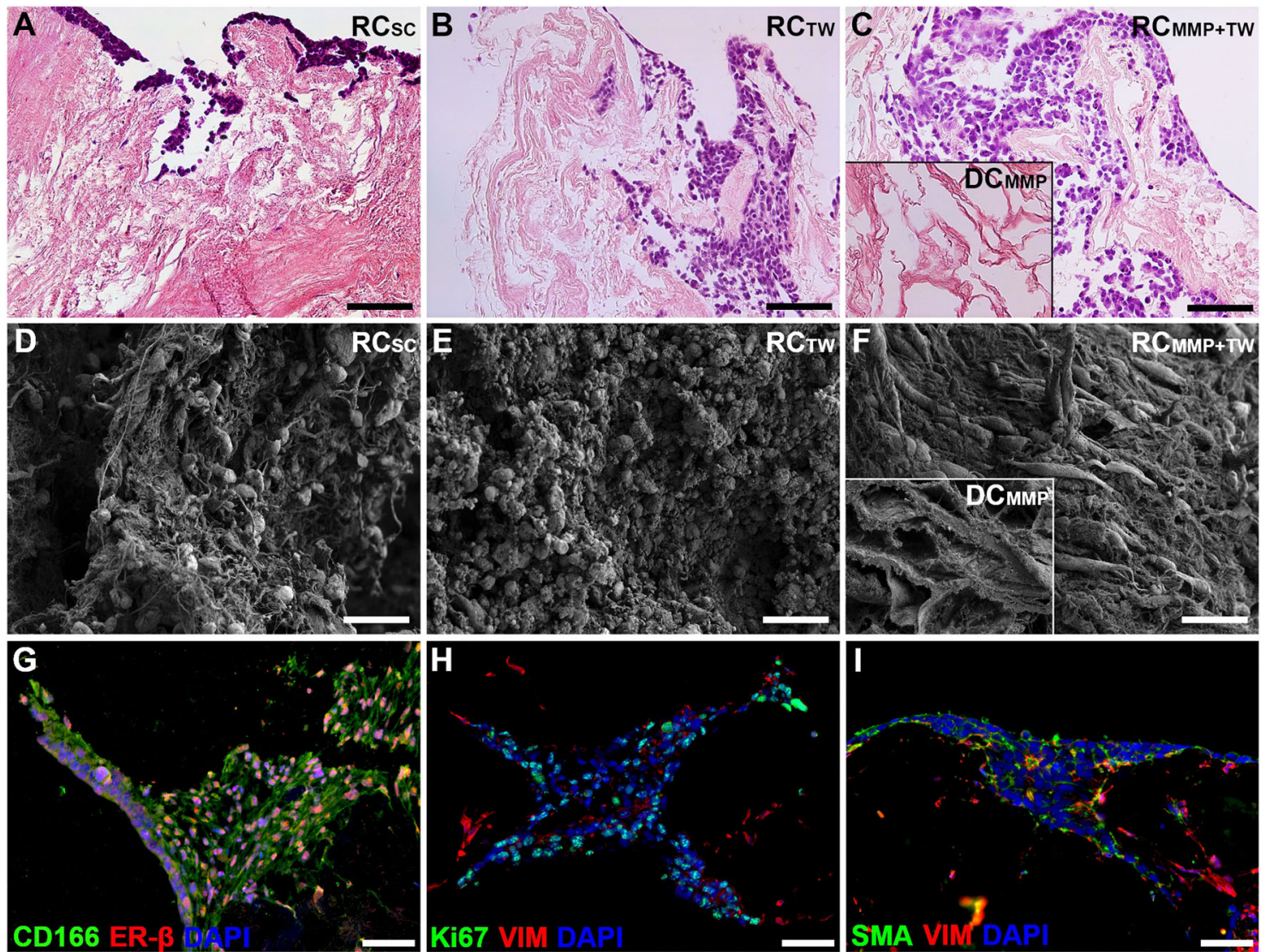

J

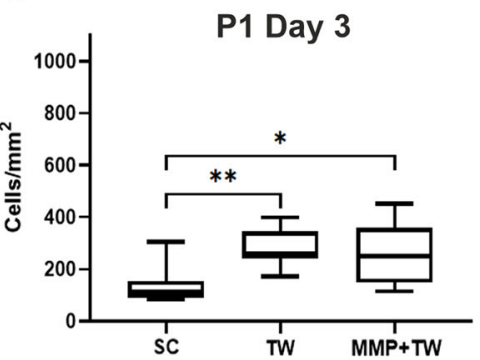

M

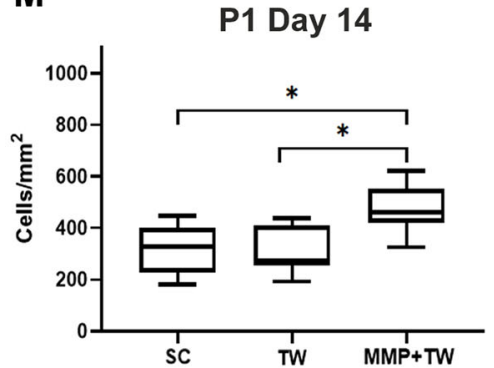

\section{K}

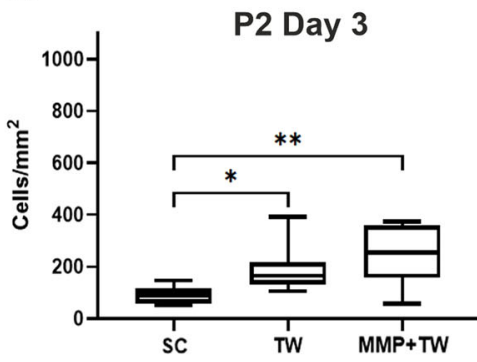

N

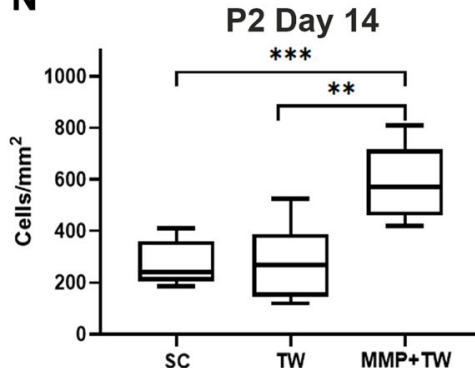

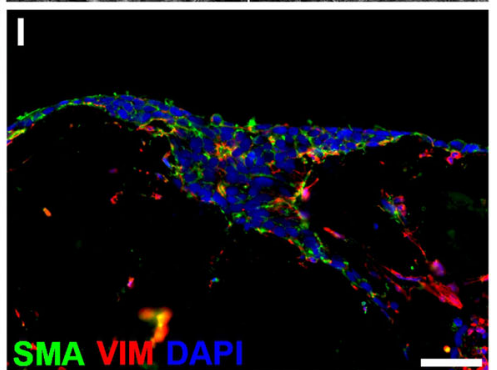

L

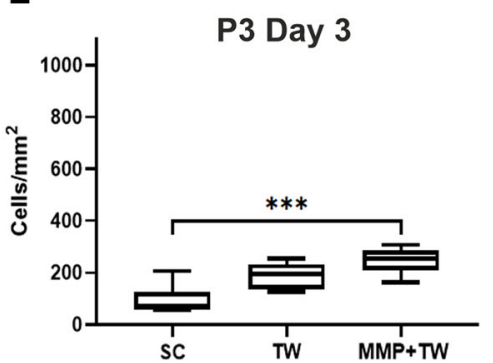

0

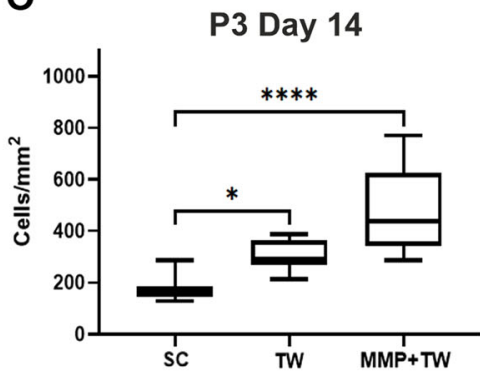

Fig. 6 Scaffold preconditioning and transwells significantly improved the recellularization effiecinecy. Representative hematoxylin and eosin-stained sections of recellularized constructs from each culturing condition after 14 days in vitro $(\mathbf{A}-\mathbf{C} ; \mathbf{s c a l e}$ bar $=100 \mu \mathrm{m})$. Cells were mainly located around the injection site when standard culturing conditions $\left(\mathrm{RC}_{\mathrm{sc}}\right)$ were used and there was limited migration $(\mathbf{A})$. However, the different recellularization strategies (transwells, $\mathrm{RC}_{\mathrm{TW}}$ ) and matrix metalloproteinase enzyme preconditioning in combination with $\mathrm{TW}$ $\left(\mathrm{RC}_{\mathrm{MMP}}+\mathrm{TW}\right)$ clearly improved the cell density and the cellular distribution throughout the scaffolding structure (B, C). Surface cell density was visualized by scanning electron microscope and showed a dense coverage, independent of strategy used (D-F; inserts in C and $\mathbf{F}$ show the scaffold preconditioned with MMPs without cells; $D C_{M M P}$ scale bar $=20 \mu \mathrm{m}$ ). Sheep fetal stem cells kept their pluripotency during recellularization independent of scaffold type, evident by the continuation of CD166, estrogen receptor- $\beta$ (ER- $\beta$ ), vimentin (VIM), $\alpha$-smooth muscle actin (SMA), and Ki67 (proliferation) expression (G-l; scale bars $=100 \mu \mathrm{m}$ ). When the recellularization efficiency was quantified and compared between groups and recellularization strategy, it became obvious that preconditioning with MMPs and using transwells were advantageous already after 3 days in vitro (J-L), but a substantial improvement was seen after 14 days (M-O) in protocol 1 (P1), protocol 2 (P2), and protocols $3(\mathrm{P} 3)$, respectively. Graphs show box plot with each sample value, median $\pm \mathrm{IQR}$, and range. Significant levels; ${ }^{*} p<0.05 ;{ }^{*} p<$ $0.01{ }^{* * *} p<0.001$, and ${ }^{* * * *} p<0.0001$. 
A.M. Padma et al.

Table 2. Statistical differences calculated based on the quantified recellularization efficiency (cell density) of different scaffold types (protocol 1, P1; protocol 2, P2; protocol 3, P3) compared with the same recellularization strategy and time point (d, days in vitro; MED, median; n.s., not significant).

\begin{tabular}{|c|c|c|c|c|c|c|c|c|}
\hline \multicolumn{3}{|c|}{ Standard conditions } & \multicolumn{3}{|l|}{ Transwell } & \multicolumn{3}{|c|}{$\mathrm{MMP}+$ transwell } \\
\hline $\mathrm{P} 1$ vs. $\mathrm{P} 2$ & n.s. & n.s. & $\mathrm{P} 1$ vs. $\mathrm{P} 2$ & $p=0.0089$ & n.s. & $\mathrm{P} 1$ vs. $\mathrm{P} 2$ & n.s. & n.s. \\
\hline $\mathrm{P} 1$ vs. P3 & n.s. & $p=0.0017$ & P1 vs. P3 & $p=0.0411$ & n.s. & $\mathrm{P} 1$ vs. P3 & n.s. & n.s. \\
\hline P2 vs. P3 & n.s. & $p=0.0156$ & P2 vs. P3 & n.s. & n.s. & P2 vs. P3 & n.s. & n.s. \\
\hline
\end{tabular}

buffer saline (PBS; $12 \mathrm{~h})$, DNase I $\left(1 \mathrm{~h} ; 37^{\circ} \mathrm{C} ; 8000 \mathrm{IU}\right.$ per organ) and then another hour with DW. This cycle was then repeated a second time. For P3, the initial detergent perfusion with SDC lasted only $4 \mathrm{~h}$, which was then followed up by the perfusion of DW (6h), Triton X-100 (12 h), and then DW $(36 \mathrm{~h})$. This cycle was then repeated a second time before the organs in this group were exposed to a $1 \mathrm{~h}$ DNase I treatment at $37^{\circ} \mathrm{C}(8000 \mathrm{IU}$ per organ). All organs from P1 to P3 were then washed with DW for an additional $48 \mathrm{~h}$, then sterilized by the perfusion of peracetic acid $(1 \mathrm{~h}, 0.1 \%$ in normal saline). The organs were then washed for $48 \mathrm{~h}$ in sterile PBS and frozen at $-20^{\circ} \mathrm{C}$ until used for the experiments mentioned below. The physical attributes of these uterus scaffolds were extensively documented in our earlier publication and were therefore not further assessed herein. To standardize each experiment below, a biopsy punch was used to create multiple full-thickness dUTDs ( $5 \mathrm{~mm}$ in diameter) that were obtained from the uterine body, a few $\mathrm{cm}$ proximal to the cervix. Thus, each dUTD contained all uterine layers. All dUTDs were then kept in PBS until used for the experiments described below.

\section{Scaffold MMPs preconditioning, DNA, protein, and hydroxyproline quantification}

About half of all the dUTDs from each scaffold type were enzymatically treated with MMP2 (2.5 $\mathrm{gg} / \mathrm{L}$, Peprotech, Stockholm, Sweden) and MMP9 $(2.5 \mu \mathrm{g} / \mathrm{L}$, Sigma-Aldrich, Gothenburg, Sweden) that had been dissolved and activated according to Sigma-Aldrich instructions using aminophenylmercuric acetate buffer. Each scaffold type was incubated at $37^{\circ} \mathrm{C}$ for $24 \mathrm{~h}$ in the MMP solution. Each disc was then washed with $20 \mathrm{mM}$ EDTA solution twice, and then with PBS.

Six dUTDs from each scaffold type, including MMPs treated, were analyzed for donor DNA content using the DNeasy Blood and Tissue Kit (\#69504; Qiagen, Sollentuna, Sweden) and a Nanodrop 2000 (Thermo Fisher Scientific, Gothenburg, Sweden). The total protein scaffold content ( $n=6$ per group) was established using the Coomassie Bradford protein assay kit as per standard protocols (\#23200; Thermo Fisher Scientific).

Hydroxyproline, the main constituent in collagen, was quantified from $100 \mathrm{mg}$ of homogenized tissue ( $n=6$ per group). Homogenates were first mixed with $\mathrm{NaOH}(10 \mathrm{~N})$ and incubated for $1 \mathrm{~h}$ at $120^{\circ} \mathrm{C}$. The hydroxyproline content was then measured using a hydroxyproline assay kit (ab222941, Abcam, Cambridge, MA) following the manufactures instructions.

\section{Histology and immunohistochemistry assessment on scaffolds}

Five $\mu \mathrm{m}$ thick tissue sections from dehydrated and paraffin-embedded MMPs treated and untreated dUTDs were mounted on slides and processed for Russel-Movat pentachrome staining using a standard staining kit (KSC-MPS-2, Nordic BioSite, Täby, Sweden). Sections from each scaffold type were also processed for immunohistochemistry. An antigen retrieval step was first conducted using citric acid $(\mathrm{pH}=6.0)$ in a pressure cooker. The sensitive Mach 3 and vulcan fast red kits (Biocare Medical, Pacheco, CA, USA) were then used to visualize collagen I (\#ab292), collagen IV (\#ab6586), laminin (\#ab11575), elastin (\#ab23748), and fibronectin (\#ab6328) using antibodies from Abcam (Cambridge, UK) at a concentration of 1:100.

\section{Vascular conduit patency test after MMPs treatment}

The Batson's \#17 plastic replica corrosion kit (Polysciences, Eppelheim, Germany) was used to investigate if the MMPs treatment may have negatively affected the patency of the vascular conduits in the scaffolds. Large scaffolds that represented half a sheep uterus with remaining vasculature were cannulated in the uterine artery and perfused with $5 \mathrm{ml}$ of the same MMP solution as used for the dUTDs. The perfused decellularized half sheep uterus was then submerged in the same solution in a $50 \mathrm{ml}$ Falcon tube and incubated at $37^{\circ} \mathrm{C}$ for $24 \mathrm{~h}$. Each scaffold was then perfused and washed with $20 \mathrm{mM}$ EDTA solution twice and then with PBS. Polymer resin from the Batson's kit was mixed according to the instructions and was then immediately injected into the uterine artery. The procedure was filmed and the files attached as supplementary information (Supplementary videos 1-3).

\section{Functional bioactivity assessment of the different uterus scaffold types}

Two bioactivity tests were conducted on each scaffold type: the DRG assay which is responsive to growth factors that stimulate axonal regeneration ${ }^{58}$, and the chicken CAM assay which is responsive to angiogenic growth factors $^{59,60}$.

The DRG assay. Bilateral DRGs were isolated from embryonic day 14 fetuses of a pregnant Wistar rat (Janvier Labs, Le Genest-Saint-Isle, France) following approved ethical guidelines (114-2014, University of Gothenburg, Sweden). Isolated DRGs were immediately placed separately on top of the endometrial side of dUTDs ( $n=8 /$ protocol). These co-cultures were incubated for 2 days in standard cell culturing medium (DMEM GlutaMAX, $1 \%$ Anti-Anti, 10\% fetal bovine serum, FBS; Thermo Fisher Scientific). DRGs cultured in collagen-coated wells with media supplemented with nerve growth factor (NGF, $10 \mathrm{ng} / \mathrm{mL}$; PeproTech, Stockholm, Sweden) were used as positive controls. Neuronal outgrowth was assessed by fluorescent immunocytochemistry on paraformaldehyde fixed co-cultures using an anti-pan neurofilament primary antibody (1:400, AB837904, Nordic Biosite, Täby, Sweden) and an Alexa Fluor 488 conjugated secondary antibody (1:300; Thermo Fisher Scientific). DNA was counterstained with $4^{\prime}, 6^{\prime}$ diamidino-2-phenylindole (DAPI, Thermo Fisher Scientific).

The chicken CAM assay. Fertilized chicken eggs (Swedfarm Väst $A B$, Herrljunga, Sweden) were placed in an egg incubator for 3 days at $37.5^{\circ} \mathrm{C}$ and then $3 \mathrm{~mL}$ of albumin was removed using a sterile $25 \mathrm{G}$ needle. This detached the CAM from the shell, and then a $1 \mathrm{~cm}^{2}$ window was cut in the shell. The opening was covered with Tegaderm film ( $3 \mathrm{M}$ Health Care, Minneapolis, Minnesota, USA) before each egg was returned to the incubator. On embryo development day (EDD) 9, the window was reopened and a dUTD was inserted onto the CAM (P1, $n=5 ; \mathrm{P} 1_{\text {MMPs, }} n=6$; $\left.\mathrm{P} 2, n=6 ; \mathrm{P}_{\mathrm{MMPs}_{1}} n=4 ; \mathrm{P} 3, n=6 ; \mathrm{P}_{\mathrm{MMPs}}, n=5\right)$. A control group was included to evaluate normal blood vessel growth by inoculating a biologically inert reference site on the CAM using a hydrogel drop of $2.2 \%$ alginate/PBS $(n=7 ; 20 \mu \mathrm{L})$. The window was again covered with Tegaderm and the egg placed back in the incubator. On EDD 14, the shell was opened further to enable photographing the implant and the embryonic vessels on the CAM under a stereomicroscope. From the photograph of each implant, two rings $(0.5-1.5 \mathrm{~mm}$ from the center) were digitally inserted in the picture at the biopsy center using ImageJ. The number of vessels was then manually counted in the area between these rings (branching vessels were counted as one) by two individuals, one of whom was blinded to the study. The inter-observer variation was insignificant, and thus, counts were averaged and used for the statistical analyses.

\section{Sheep fetal stem cell isolation and characterization}

Heterogeneous SF-SCs were isolated from the femurs of a 6-8-week-old sheep fetus (obtained at the local abattoir) by flushing with L-15 medium supplemented with Anti-Anti (Thermo Fisher Scientific). Cells were then expanded in vitro and verified for multipotency according to differentiation methods for chondrogenesis ${ }^{61}$, osteogenesis ${ }^{62}$, and myogenesis ${ }^{63}$. 
Differentiated and undifferentiated SF-SCs were fixed in $4 \%$ buffered formaldehyde and stained with a wide range of primary antibodies $(1 \mathrm{~h}$ at room temperature) from Abcam (Amsterdam, Netherlands), including aSMA (ab32575; 1:500), vimentin (ab8798; 1:100), CD166 (ab235957; 1:200), Ki67 (ab15580; 1:300), estrogen receptor-a (ER-a; ab66102; 1:100), ER- $\beta$ (ab187291; 1:100), progesterone receptor (PR; ab2765; 1:100), cytokeratin (ab9377; 1:1000), MyoD1 (ab16148, 1:100), RANK (ab13918, 1:100), and DMP1 (ab103203, 1:100). Each primary antibody was conjugated with either CY3 or Alexa Fluor 488 secondary antibodies (Thermo Fisher; 1:300) and DNA was labeled with DAPI. Chondrogenesis was evaluated after alcian blue staining using standard methods.

\section{Recellularization using standard cell culturing conditions}

The dUTDs were recellularized with SF-SCs by injecting each disc $(n=20$ per scaffold type) with a total of $10^{6}$ cells in 10 repeated injections using a $30 \mathrm{G}$ needle. The recellularized scaffolds were cultured with the endometrial side positioned upwards using standard cell culturing conditions with medium changed every second day. This group was named $\mathrm{RC}_{\mathrm{sC}}$.

\section{Recellularization using transwell inserts}

Additional dUTDs ( $n=20$ per scaffold type) were placed individually on transwell inserts ( $0.4 \mu \mathrm{m}$, Sarstedt, Nümbrecht, Germany) in a 24-well plate and were then completely covered with cell culture medium. The transwell inserts improved cell culture medium access to the bottom (myometrial side) of the recellularized scaffold during culture and thereby also the nutritional and metabolite exchange in the deeper compartment of the scaffolds. The cell injections were conducted the same way as mentioned above. This group was named $\mathrm{RC}_{\mathrm{TW}}$.

\section{Recellularization of enzymatically preconditioned scaffolds in transwell inserts}

Twenty dUTDs per scaffold type were placed individually in transwell inserts and recellularized and cultured as earlier mentioned. This group was named $\mathrm{RC}_{\mathrm{MMP}+\mathrm{TW}}$

\section{Histology, immunohistochemistry, and cell counting on recellularized scaffolds}

The recellularization efficiency of each construct was evaluated on day 3 and day 14 post recellularization day by histology using standard hematoxylin \& eosin (H\&E) and DAPI staining protocols $(n=9$ per scaffold type, per recellularization method, and per time point). In addition, some sections were stained with the same antibodies that were used to characterize the cells in vitro, using the same methods but with an additional antigen retrieval step using citrate buffer. The recellularization efficiency (cells $/ \mathrm{mm}^{2}$ ) was based on the total number of DAPI-positive cells found in six randomly photographed fields from each recellularized dUTD $\left(\times 200\right.$ magnification; photo area $\left.=0.776 \mathrm{~mm}^{2}\right)$. The total cell number was quantified using ImageJ, then the value transformed to number of cells/ $\mathrm{mm}^{2}$, and was then averaged for each group, recellularization strategy, scaffold type, and time point after recellularization.

\section{Scanning electron microscopy}

Samples assessed by scanning electron microscopy included discs of decellularized-, MMPs treated-, and recellularized tissue. Each specimen was prepared using the osmium tetroxide and thiocarbohydrazide method $^{64}$, and was imaged with Zeiss Gemini-2 SEM 500 (Carl Zeiss AG, Oberkochen, Germany). The quantification of collagen fiber thickness was performed on images taken at $\times 50,000$ magnification. Each image was opened in ImageJ, the scale was set and the measuring tool in the software was used to measure the width of 25 individual collagen fiber strands in each image.

\section{Statistics}

Statistics was performed using Graphpad Prism (Graphpad, CA, USA). All data sets were tested for normality using the Shapiro-Wilk test. Parametric data were assessed with one-way ANOVA and Tukey's HSD post hoc test and presented with bar graphs (mean \pm standard error of mean, SEM). Non-parametric data were presented in box plots with median, interquartile range, and range. Kruskal-Wallis test was conducted to evaluate differences (corrected by Dunn's post hoc test for multiple group comparisons). Mann-Whitney U-test was used to compare differences between the recellularized constructs after 3 and 14 days in vitro for the same scaffold type and culturing condition.

\section{Reporting summary}

Further information on research design is available in the Nature Research Reporting Summary linked to this article.

\section{DATA AVAILABILITY}

Original data generated for this article are available from the corresponding author on reasonable request.

Received: 6 October 2020; Accepted: 20 April 2021; Published online: 21 May 2021

\section{REFERENCES}

1. Brännström, M. et al. Livebirth after uterus transplantation. Lancet $\mathbf{3 8 5}, 607-616$ (2015).

2. Brucker, S. Y. et al. Living-donor uterus transplantation: pre-, intra-, and postoperative parameters relevant to surgical success, pregnancy, and obstetrics with live births. J. Clin. Med. 9, 2485 (2020).

3. Brännström, M. Uterus transplantation. Curr. Opin. Organ Transpl. 20, 621-628 (2015).

4. Brännström, M. et al. One uterus bridging three generations: first live birth after mother-to-daughter uterus transplantation. Fertil. Steril. 106, 261-266 (2016).

5. Castellon, L. A. R. et al. The history behind successful uterine transplantation in humans. JBRA Assist. Reprod. 21, 126-134 (2017).

6. Ejzenberg, D. et al. Livebirth after uterus transplantation from a deceased donor in a recipient with uterine infertility. Lancet 392, 2697-2704 (2019).

7. Fronek, J. et al. Live birth following uterine transplantation from a nulliparous deceased donor. Transplantation 105, 1077-1081 (2020).

8. Jones, B. P. et al. Human uterine transplantation: a review of outcomes from the first 45 cases. BJOG 126, 1310-1319 (2019).

9. Testa, G. et al. First live birth after uterus transplantation in the United States. Am. J. Transpl. 18, 1270-1274 (2018).

10. Padma, A. M. et al. The development of an extended normothermic ex vivo reperfusion model of the sheep uterus to evaluate organ quality after cold ischemia in relation to uterus transplantation. Acta Obstet. Gynecol. Scand. 98 1127-1138 (2019).

11. Arantes, R. M. et al. Novel technique in a sheep model of uterine transplantation. Transpl. Proc. 52, 1399-1401 (2020).

12. Kisu, I. et al. A surgical technique using the ovarian vein in non-human primate models of potential living-donor surgery of uterus transplantation. Acta Obstet. Gynecol. Scand. 94, 942-948 (2015).

13. Brännström, M. et al. Live birth after robotic-assisted live donor uterus transplantation. Acta Obstet. Gynecol. Scand. 99, 1222-1229 (2020).

14. Falcone, T. \& Richards, E. G. Balancing innovation and safety: robotic-assisted donor surgery in uterus transplantation. Fertil. Steril. 114, 976-977 (2020).

15. Fornalik, H. \& Fornalik, N. Uterus transplantation:robotic surgeon perspective. Fertil. Steril. 109, 365 (2018).

16. Ding, L. et al. Transplantation of bone marrow mesenchymal stem cells on collagen scaffolds for the functional regeneration of injured rat uterus. Biomaterials 35, 4888-4900 (2014).

17. Kniazeva, E. et al. Primordial follicle transplantation within designer biomaterial grafts produce live births in a mouse infertility model. Sci. Rep. 5, 17709 (2015).

18. Londra, L., Chuong, F. S. \& Kolp, L. Mayer-Rokitansky-Kuster-Hauser syndrome: a review. Int. J. Women's Health 7, 865-870 (2015).

19. Luyckx, V. et al. A new step toward the artificial ovary: survival and proliferation of isolated murine follicles after autologous transplantation in a fibrin scaffold. Fertil. Steril. 101, 1149-1156 (2014).

20. Vanacker, J. et al. Transplantation of an alginate-matrigel matrix containing isolated ovarian cells: first step in developing a biodegradable scaffold to transplant isolated preantral follicles and ovarian cells. Biomaterials 33, 6079-6085 (2012).

21. Hiraoka, T. et al. STAT3 accelerates uterine epithelial regeneration in a mouse model of decellularized uterine matrix transplantation. JCl Insight. 1, e87591, https://doi.org/10.1172/jci.insight.87591 (2016).

22. Hellström, M. et al. Towards the development of a bioengineered uterus: comparison of different protocols for rat uterus decellularization. Acta Biomater. 10 5034-5042 (2014). 
23. Miki, F. et al. The orientation of a decellularized uterine scaffold determines the tissue topology and architecture of the regenerated uterus in rats. Biol. Reprod. 100, 1215-1227 (2019).

24. Miyazaki, K. \& Maruyama, T. Partial regeneration and reconstruction of the rat uterus through recellularization of a decellularized uterine matrix. Biomaterials 35, 8791-8800 (2014).

25. Padma, A. M. et al. Protocols for rat uterus isolation and decellularization: applications for uterus tissue engineering and 3D cell culturing. Methods Mol. Biol. 1557, 161-175 (2017).

26. Santoso, E. G. et al. Application of detergents or high hydrostatic pressure as decellularization processes in uterine tissues and their subsequent effects on in vivo uterine regeneration in murine models. PLOS ONE 9, e103201 (2014).

27. Campo, H. et al. Tissue-specific decellularized endometrial substratum mimicking different physiological conditions influences in vitro embryo development in a rabbit model. Acta Biomater. 89, 126-138 (2019).

28. Campo, H. et al. De- and recellularization of the pig uterus: a bioengineering pilot study. Biol. Reprod. 96, 34-45 (2017).

29. Daryabari, S. S. et al. Development of an efficient perfusion-based protocol for whole-organ decellularization of the ovine uterus as a human-sized model and in vivo application of the bioscaffolds. J. Assist. Reprod. Genet. 36, 1211-1223 (2019).

30. Tiemann, T. T. et al. Towards uterus tissue engineering: a comparative study of sheep uterus decellularisation. Mol. Hum. Reprod. 26, 257-270 (2020).

31. Hellström, M. et al. Bioengineered uterine tissue supports pregnancy in a rat model. Fertil. Steril. 106, 487-96.e1 (2016).

32. Couri, B. M. et al. Animal models of female pelvic organ prolapse: lessons learned. Expert Rev. Obstet. Gynecol. 7, 249-260 (2012).

33. Krause, H. \& Goh, J. Sheep and rabbit genital tracts and abdominal wall as an implantation model for the study of surgical mesh. J. Obstet. Gynaecol. Res. 35, 219-224 (2009).

34. Dorin, R. P., Pohl, H. G., De Filippo, R. E., Yoo, J. J. \& Atala, A. Tubularized urethral replacement with unseeded matrices: what is the maximum distance for normal tissue regeneration? World J. Urol. 26, 323-326 (2008).

35. Howard, D., Buttery, L. D., Shakesheff, K. M. \& Roberts, S. J. Tissue engineering: strategies, stem cells and scaffolds. J. Anat. 213, 66-72 (2008).

36. L'Heureux, N., McAllister, T. N. \& de la Fuente, L. M. Tissue-engineered blood vessel for adult arterial revascularization. N. Engl. J. Med. 357, 1451-1453 (2007).

37. Ochando, J., Charron, D., Baptista, P. M. \& Uygun, B. E. Immune responses to bioengineered organs. Curr. Opin. Organ Transpl. 22, 79-85 (2017).

38. Kim, J. et al. Fabrication of uterine decellularized matrix using high hydrostatic pressure through depolymerization of actin filaments. J. Biomech. Sci. Eng. 14, 1900097-19 (2019).

39. Hellström, M., Bandstein, S. \& Brännström, M. Uterine tissue engineering and the future of uterus transplantation. Ann. Biomed. Eng. 45, 1718-1730 (2017)

40. Shen, Y. Y. et al. Biological characteristics of dynamic expression of nerve regeneration related growth factors in dorsal root ganglia after peripheral nerve injury. Neural Regen. Res. 15, 1502-1509 (2020).

41. Dubovy, P., Hradilova-Svizenska, I., Klusakova, I., Brazda, V. \& Joukal, M. Interleukin- 6 contributes to initiation of neuronal regeneration program in the remote dorsal root ganglia neurons after sciatic nerve injury. Histochem Cell Biol. 152, 109-117 (2019).

42. Newberry, K. et al. Development of a spontaneously active dorsal root ganglia assay using multiwell multielectrode arrays. J. Neurophysiol. 115, 3217-3228 (2016).

43. Mastrullo, V., Cathery, W., Velliou, E., Madeddu, P. \& Campagnolo, P. Angiogenesis in tissue engineering: as nature intended? Front Bioeng. Biotechnol. 8, 188 (2020).

44. Ott, H. C. et al. Regeneration and orthotopic transplantation of a bioartificial lung. Nat. Med. 16, 927-933 (2010).

45. Ott, H. C. et al. Perfusion-decellularized matrix: using nature's platform to engineer a bioartificial heart. Nat. Med. 14, 213-221 (2008).

46. Shimoda, H. et al. Decellularized liver scaffolds promote liver regeneration after partial hepatectomy. Sci. Rep. 9, 12543 (2019)

47. Pors, S. E. et al. Initial steps in reconstruction of the human ovary: survival of preantral stage follicles in a decellularized human ovarian scaffold. Hum. Reprod. 34, 1523-1535 (2019).

48. Wang, M. et al. Immobilization of heparin on decellularized kidney scaffold to construct microenvironment for antithrombosis and inducing reendothelialization. Sci. China Life Sci. 61, 1168-1177 (2018).

49. Eivazkhani, F. et al. Evaluating two ovarian decellularization methods in three species. Mater. Sci. Eng. C. Mater. Biol. Appl. 102, 670-682 (2019).

50. Schneeberger, K. et al. Large-scale production of LGR5-positive bipotential human liver stem cells. Hepatology 72, 257-270 (2019).

51. Mirzaeian, L. et al. Optimizing the cell seeding protocol to human decellularized ovarian scaffold: application of dynamic system for bio-engineering. Cell J. 22, 227-235 (2020).
52. Elebring, E., Kuna, V. K., Kvarnström, N. \& Sumitran-Holgersson, S. Cold-perfusion decellularization of whole-organ porcine pancreas supports human fetal pancreatic cell attachment and expression of endocrine and exocrine markers. $J$. Tissue Eng. 8, 2041731417738145 (2017).

53. Amable, P. R., Teixeira, M. V., Carias, R. B., Granjeiro, J. M. \& Borojevic, R. Protein synthesis and secretion in human mesenchymal cells derived from bone marrow, adipose tissue and Wharton's jelly. Stem Cell Res. Ther. 5, 53 (2014).

54. Lozito, T. P., Jackson, W. M., Nesti, L. J. \& Tuan, R. S. Human mesenchymal stem cells generate a distinct pericellular zone of MMP activities via binding of MMPs and secretion of high levels of TIMPs. Matrix Biol. 34, 132-143 (2014).

55. Forsyth, P. A. et al. Gelatinase-A (MMP-2), gelatinase-B (MMP-9) and membrane type matrix metalloproteinase-1 (MT1-MMP) are involved in different aspects of the pathophysiology of malignant gliomas. Br. J. Cancer 79, 1828-1835 (1999).

56. Weiss, A. R. R. \& Dahlke, M. H. Immunomodulation by mesenchymal stem cells (MSCs): mechanisms of action of living, apoptotic, and dead MSCs. Front Immunol. 10, 1191 (2019).

57. Santamaria, X. et al. Autologous cell therapy with CD133+ bone marrow-derived stem cells for refractory Asherman's syndrome and endometrial atrophy: a pilot cohort study. Hum. Reprod. 31, 1087-1096 (2016).

58. $\mathrm{Hu}, \mathrm{Y}$. et al. Lentiviral-mediated transfer of CNTF to schwann cells within reconstructed peripheral nerve grafts enhances adult retinal ganglion cell survival and axonal regeneration. Mol. Ther. 11, 906-915 (2005).

59. $\mathrm{Li}, \mathrm{M}$. et al. The in ovo chick chorioallantoic membrane (CAM) assay as an efficient xenograft model of hepatocellular carcinoma. J. Vis. Exp. 104, e52411 (2015).

60. Sys, G. M. et al. The in ovo CAM-assay as a xenograft model for sarcoma. J. Vis. Exp. 77, e50522 (2013)

61. Solchaga, L. A., Penick, K. J. \& Welter, J. F. Chondrogenic differentiation of bone marrow-derived mesenchymal stem cells: tips and tricks. Methods Mol. Biol. 698, 253-278 (2011)

62. Liao, H. T. \& Chen, C. T. Osteogenic potential: comparison between bone marrow and adipose-derived mesenchymal stem cells. World J. Stem Cells 6, 288-295 (2014).

63. Galli, D., Vitale, M. \& Vaccarezza, M. Bone marrow-derived mesenchymal cell differentiation toward myogenic lineages: facts and perspectives. Biomed. Res. Int. 2014, 762695 (2014).

64. Davies, S. \& Forge, A. Preparation of the mammalian organ of Corti for scanning electron microscopy. J. Microsc. 147, 89-101 (1987).

\section{ACKNOWLEDGEMENTS}

The authors thank Ingigerdur Gudmundsdottir for assistance during the revision process. The study was financed by the Knut and Alice Wallenberg Foundation, the Swedish research council (VR: 116008), the ALF-agreement (between the Swedish government and the county council), the Adlerbertska, Hjalmar Svensson, and Wilhelm \& Martina Lundgrens research foundations, the Swedish-Korean International Cooperation in Research and Higher Education (STINT) and the International Research and Development Program of the NRF of Korea, funded by the Ministry of Science and ICT of Korea (NRF-2018K2A9A2A12000216) with additional funding from NRF-2018R1D1A1B07049059. We thank CCl at University of Gothenburg and the NMI (VR-RFI 2016-00968) for assistance in electron microscopy.

\section{AUTHOR CONTRIBUTIONS}

A.M.P., L.C., F.K.K., E.S., S.B. and T.T.T. conducted most of the practical work with assistance and supervision from M.O., M.J.S. and M.H. A.M.P., L.C., M.J.S., M.O., M.B. and M.H. designed the project. A.M.P., M.B. and M.H. drafted the manuscript. All authors contributed to the interpretation of data, concluding the results, and improving the manuscript to its final version.

\section{FUNDING}

Open access funding provided by University of Gothenburg.

\section{COMPETING INTERESTS}

The authors declare no competing interests.

\section{ADDITIONAL INFORMATION}

Supplementary information The online version contains supplementary material available at https://doi.org/10.1038/s41536-021-00136-0.

Correspondence and requests for materials should be addressed to M.H. 
Reprints and permission information is available at http://www.nature.com/ reprints

Publisher's note Springer Nature remains neutral with regard to jurisdictional claims in published maps and institutional affiliations.

(i) Open Access This article is licensed under a Creative Commons Attribution 4.0 International License, which permits use, sharing, adaptation, distribution and reproduction in any medium or format, as long as you give appropriate credit to the original author(s) and the source, provide a link to the Creative Commons license, and indicate if changes were made. The images or other third party material in this article are included in the article's Creative Commons license, unless indicated otherwise in a credit line to the material. If material is not included in the article's Creative Commons license and your intended use is not permitted by statutory regulation or exceeds the permitted use, you will need to obtain permission directly from the copyright holder. To view a copy of this license, visit http://creativecommons. org/licenses/by/4.0/.

(c) The Author(s) 2021 